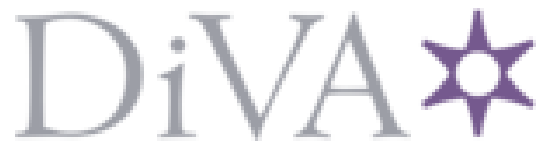

http://www.diva-portal.org

Preprint

This is the submitted version of a paper published in .

Citation for the original published paper (version of record):

Arvidsson, M., Ringstad, L., Skedung, L., Duvefelt, K., Rutland, M W. (2017)

Feeling fine - the effect of topography and friction on perceived roughness and slipperiness

Biotribology, 11: 92-101

https://doi.org/10.1016/j.biotri.2017.01.002

Access to the published version may require subscription.

N.B. When citing this work, cite the original published paper.

Permanent link to this version:

http://urn.kb.se/resolve?urn=urn:nbn:se:ri:diva-31183 


\title{
Feeling fine - the effect of topography and friction on perceived roughness and slipperiness
}

\author{
Martin Arvidsson ${ }^{1}$, Lovisa Ringstad ${ }^{1}$, Lisa Skedung ${ }^{1}$, Kenneth Duvefelt $^{2}$ \& Mark W Rutland ${ }^{1,3}$ \\ ${ }^{1}$ SP Technical Research Institute of Sweden, Chemistry, Materials and Surfaces, Box 5607, SE-114 86 Stockholm, \\ Sweden \\ ${ }^{2}$ Machine Design, KTH Royal Institute of Technology, Brinellvägen 83, SE-100 44 Stockholm, Sweden \\ ${ }^{3}$ Surface and corrosion science, KTH Royal Institute of Technology, Drottning Kristinas Väg 51, SE-100 44 Stockholm, \\ Sweden \\ Corresponding: Mark W Rutland \\ E-mail: mark@kth.se
}

\begin{abstract}
(1) Background. To design materials with specific haptic qualities, it is important to understand both the contribution of physical attributes from the materials surfaces and the perceptions that are involved in the haptic interaction. (2) Methods. A series of 16 wrinkled surfaces consisting of two similar materials, of different elastic modulus and 8 different wrinkle wavelengths were thus characterized in terms of surface roughness and tactile friction coefficient. Sixteen participants scaled the perceived Roughness and Slipperiness of the surfaces using free magnitude estimation. Friction experiments were performed both by participants and by a trained experimenter with higher control. (3) Results and discussion. The trends in friction properties were similar for the group of participants performing the friction measurements in an uncontrolled way and the experiments performed under well-defined conditions, showing that the latter type of measurements represent the general friction properties well. The results point to slipperiness as the key perception dimension for textures below $100 \mu \mathrm{m}$ and roughness above $100 \mu \mathrm{m}$. Furthermore, it is apparent that roughness and slipperiness perception of these types of structures are not independent. The friction is related to contact area between finger and material. Somewhat surprising was that the material with the higher elastic modulus was perceived as more slippery. A concluding finding was that the flat (high friction) references surfaces were scaled as rough, supporting the theory that perceived roughness itself is a multidimensional construct with both surface roughness and friction components.
\end{abstract}

\section{Keywords}

Tactile perception, roughness, slipperiness, friction 


\section{Introduction}

Tactile perception is considered an important contributor to the overall experience of products such as packaging, magazines, fabrics, skin and hair care products and tactile displays. Predictive capacity of how new products or modifications of existing products will be tactually perceived by the customer or end user, would facilitate product development and make it more cost-effective, due to a more systematic product design with less trial and error. Thus, an understanding of how process parameters, material and surface properties (e.g. surface roughness, thermal conductivity and diffusivity) and interfacial phenomena such as friction affect the perceptual response is of importance.

Active exploration of a surface with a human hand is really a tribological event and consequently the tactile interest has increased rapidly over the last years within the field of tribology [1-3]. The friction that arises during active exploration at the finger-surface interface is sometimes called tactile friction [3-5]. From a perception perspective it is preferable to mimic the haptic interrogation process by moving the finger over a stationary surface placed on a force cell device $[2,6-17]$. The skin friction and skin mechanics of this situation has recently been reviewed $[3,18,19]$. The friction response is highly affected by various properties such as finger hydration $[5,7,17,20,21]$ and surface roughness $[4,5,7,22,23]$. Typically friction decreases with increasing surface roughness at the small scale and the trend reverses at higher roughness. A common feature of skin friction studies is the variation in the response between different participants, which is often associated with variations in skin moisture content [20, 22, 24, 25]. While skin friction has been addressed extensively, less effort has been made to combine tactile friction measurements and surface properties with perceptual evaluation, i.e. measurements with human subjects. Derler and Gerhard [19] observe that tactile perception and haptics in relation to skin tribology is largely unstudied and poorly understood. Liu et al. [26] correlated tactile friction and surface roughness with the roughsmooth and grippy-slippery perceptions on car seat materials and Barnes et al [27] found that glass surfaces were perceived pleasant or desirable once the surface was less rough than the fingertip. Chen et al [28] investigated relations between touch perceptions of different cardboards and physical measurements. Questionnaires of warmth-cold, slippery-sticky, smooth-rough, hard-soft, bumpy-flat and wet-dry were linked with physical measurements of surface roughness, compressibility, friction and the rate of cooling and the touch perception was to be associated with more than physical property. Chen et al [29] also investigated relationships between the affective and sensorial judgments and the various physical measurements and concluded that further work is needed, particularly to quantify roughness and sliding friction in a manner useful for relating to the affective responses.

In order to get a more comprehensive picture of tactile perception, researchers have tried to map the dimensions of the tactile space using multidimensional scaling [30-35]. Texture perception is generally found to be 3-dimensional with a roughness-smoothness, softness-hardness, and a stickiness-slipperiness dimension [30, 31, 33]. The dimensionality has also been found to be similar in micro-textures with structures below $100 \mu \mathrm{m}[36,37]$.

The sense of touch is quite remarkable in detecting small textural variations. Single asperities down to at least $1 \mu \mathrm{m}$ can be detected [38] and applying a repeated wave pattern with an amplitude of approximately $10 \mathrm{~nm}$ is enough to change the perception from that of a smooth surface with otherwise identical chemistry [10]. Texture perception has attracted a lot of attention in recent years and the majority of effort has 
focused on roughness, e.g. [39]. While roughness of coarse surfaces is relatively well understood, the same cannot be said for roughness of finer surfaces. There are different theories of how the neural coding of roughness of fine textures work. Some propose a spatial (SA1) model for roughness coding [40-42] where SA1 afferents code roughness for all textures. Others propose a duplex theory where SA1 afferents code roughness above $200 \mu \mathrm{m}$ and Pacinian corpuscles (PC) below $200 \mu \mathrm{m}$, through vibrations, [33, 43, 44], both with compelling arguments. It has also been proposed that spatial information was retrievable well below the postulated limit for the SA1 afferents, through vibrations[10, 45]. This notion was given some support by Weber, et al [46] who found that both temporal and spatial codes through Rapidly Adapting (RA) and PC afferents were indeed used in haptic perception of micro-textures, this idea was further elaborated on by Saal and Bensmaïa [47]. As spatial information gets more difficult to rely on, vibrations and friction become more significant in texture perception. Though slipperiness is much less studied than roughness [39], friction has been found to be the main physical property associated with slipperiness perception, e.g. [48]. In a recent study on haptic perception of micro-textures, two dimensions were found to be sufficient to explain the similarities in $0-80 \mu \mathrm{m}$ wavelength surfaces and were identified with the physical parameters of friction and wavelength [10]. What was not clear from that study was the relationship between slipperiness and roughness. Here we have thus examined the relationship between perceived roughness and perceived slipperiness as well as the psychophysical relationship with friction and topography for micro-textures.

\section{Materials and methods}

\subsection{Fabrication and characterisation of wrinkle-patterned surfaces}

Model surfaces with controlled texture were prepared by surface wrinkling or 3D-printing. The surface wrinkling procedure, used to obtain structures in the wavelength region 30-100 $\mu \mathrm{m}$, has been described in detail previously $[10,49,50]$. In brief, polydimethylsiloxane (PDMS, Sylgard 184 Dow Corning, USA) was stretched and then exposed to ultraviolet ozone (UVO) (UV/Ozone ProCleaner, Bioforce Nanosciences), oxidizing a thin overlayer of the PDMS into a stiffer film with higher elastic modulus than the rest of the substrate. When the strain was released surface wrinkles formed spontaneously due to the difference in elastic modulus between the layers [50]. By altering the stretch distance (\% increase compared to the original substrate) and UVO exposure time the desired structures were obtained, as summarized in Table 1. Surfaces with wavelengths above $100 \mu \mathrm{m}$ were obtained by 3D-printing (3D-Intelligence, Grästorp, Sweden) on VeroClear-RGD810 (an acrylic based photopolymer) followed by replication onto PDMS. Each structured PDMS-substrate was then replicated onto less rubbery materials. The materials used were two different UV-curable adhesive polymers with different elasticity, NOA73 (Norland Optical Adhesive 73, Shore D 60, Young's modulus: $11 \mathrm{MPa}$, Norland Products Inc., Cranbury, USA) and NOA81 (Norland Optical Adhesive 81, Shore D 90, Young's modulus: 1379 MPa, Norland Products Inc., Cranbury, USA). Approximately $1.1 \mathrm{~g}$ of the adhesive polymer was placed on the PDMS substrate whereupon a glass slide 
was placed on top of the polymer layer. Care was taken to avoid air bubbles. The surfaces were then cured under a UV-lamp (Original Philips UVA, $\lambda=315-400 \mathrm{~nm}$ ) for 20 minutes.

The wrinkle amplitude and wavelength of the surfaces was measured with a stylus profilometer (Taylor Hobson Form Talysurf PGI 800). The diamond stylus tip with a radius of $2 \mu \mathrm{m}$ was drawn $45 \mathrm{~mm}$ perpendicular to the wrinkled surfaces at a speed of $0.5 \mathrm{~mm} / \mathrm{s}$. For all surfaces the topography of the surfaces was scanned along three positions of the sample and all wavelength and amplitude values are averages from these three positions. The wrinkle wavelength was estimated from the average spacing between adjacent peaks (RS), and the wrinkle amplitude was estimated from the average vertical separation of the five highest peaks and the five lowest valleys ( $\mathrm{Rz} / 2)$, In addition, 3D-profiles were measured on a selection of the surfaces by scanning an area of $1 \times 1 \mathrm{~mm}$ with a resolution of $0.25 \mu \mathrm{m}$ in the $x$-direction and $10 \mu \mathrm{m}$ in the $y$-direction (Figure 1). The amplitude and wavelength were measured directly after manufacturing the surfaces and again after completing the psychophysical and tactile friction studies in order to verify that the structures were maintained after extensive usage. In total, 14 patterned surfaces and 2 un-patterned reference materials were used as stimuli in psychophysical experiments and in tactile friction measurements. The surface characteristics of these surfaces before and after use are given in Table 1, and 3D images of surface S8 and S14 are presented in Figure 1.
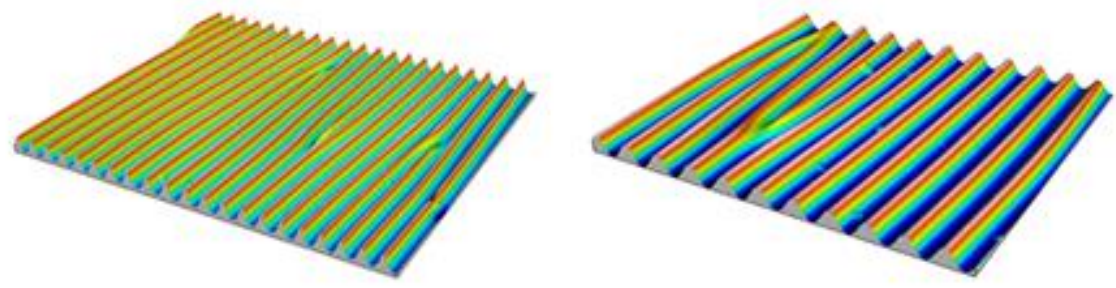

Figure 1. 3D images of surfaces S8 (left) and S14 (right) recorded after completing the perception study. Area: $1 \times 1 \mathrm{~mm}$. The wavelength and amplitude of S8 are $50 \mu \mathrm{m}$ and $4 \mu \mathrm{m}$, respectively. For S14 the wavelength and amplitude are $96 \mu \mathrm{m}$ and $6.4 \mu \mathrm{m}$, respectively. Information on wavelength and amplitude for all surfaces used in the study are presented in Table 1. 
Table 1. Properties of the model surfaces. The wavelength $(\lambda)$ and amplitude $(A)$ before and after use in friction and perception studies was measured by profilometry. The preparation parameters - stretch distance and UVO irradiation time- are also given.

\begin{tabular}{|c|c|c|c|c|c|c|c|}
\hline ID & $\lambda_{\text {before }}(\mu \mathrm{m})$ & $A_{\text {before }}(\mu \mathrm{m})$ & Material & $\begin{array}{l}\text { Prep. } \\
\text { Method }\end{array}$ & $\begin{array}{l}\text { Prep. } \\
\text { Settings } \\
\text { prep. }\end{array}$ & $\lambda_{\text {after }}(\mu \mathrm{m})$ & $\mathbf{A}_{\text {after }}(\mu \mathrm{m})$ \\
\hline S1 & - & - & NOA 73 & $\begin{array}{l}\text { None, } \\
\text { reference }\end{array}$ & - & - & - \\
\hline S2 & - & - & NOA 81 & $\begin{array}{l}\text { None, } \\
\text { reference }\end{array}$ & - & - & - \\
\hline S3 & $30.5 \pm 0.5$ & $1.7 \pm 0.1$ & NOA 73 & Wrinkling & $\begin{array}{l}50 \% \\
80 \mathrm{~min}\end{array}$ & $30.4 \pm 0.8$ & $1.6 \pm 0.1$ \\
\hline S4 & $30.5 \pm 0.8$ & $1.7 \pm 0.1$ & NOA 81 & Wrinkling & $\begin{array}{l}50 \%, \\
80 \mathrm{~min}\end{array}$ & $30.5 \pm 0.8$ & $1.6 \pm 0.03$ \\
\hline S5 & $42.5 \pm 0.4$ & $3.4 \pm 0.3$ & NOA 73 & Wrinkling & $\begin{array}{l}30 \%, \\
120 \text { min }\end{array}$ & $42.5 \pm 0.4$ & $3.4 \pm 0.2$ \\
\hline S6 & $42.6 \pm 0.3$ & $3.6 \pm 0.3$ & NOA 81 & Wrinkling & $\begin{array}{l}30 \%, \\
120 \text { min }\end{array}$ & $42.9 \pm 0.3$ & $3.6 \pm 0.3$ \\
\hline S7 & $52.0 \pm 2.7$ & $4.0 \pm 0.4$ & NOA 73 & Wrinkling & $\begin{array}{l}40 \%, \\
160 \text { min }\end{array}$ & $50.3 \pm 1.6$ & $4.0 \pm 0.4$ \\
\hline S8 & $50.2 \pm 1.4$ & $4.1 \pm 0.3$ & NOA 81 & Wrinkling & $\begin{array}{l}40 \%, \\
160 \text { min }\end{array}$ & $52.5 \pm 4.6$ & $3.8 \pm 0.7$ \\
\hline S9 & $58.4 \pm 1.7$ & $6.8 \pm 0.5$ & NOA 73 & Wrinkling & $\begin{array}{l}50 \%, \\
160 \text { min }\end{array}$ & $58.0 \pm 2.6$ & $6.6 \pm 0.4$ \\
\hline S10 & $58.2 \pm 1.9$ & $7.3 \pm 0.1$ & NOA 81 & Wrinkling & $\begin{array}{l}50 \%, \\
160 \text { min }\end{array}$ & $58.6 \pm 2.2$ & $7.1 \pm 0.1$ \\
\hline S11 & $79.4 \pm 1.5$ & $8.0 \pm 0.4$ & NOA 73 & Wrinkling & $\begin{array}{l}30 \%, \\
240 \min \end{array}$ & $79.1 \pm 0.9$ & $8.1 \pm 0.1$ \\
\hline S12 & $79.0 \pm 2.3$ & $8.4 \pm 0.4$ & NOA 81 & Wrinkling & $\begin{array}{l}30 \%, \\
240 \mathrm{~min}\end{array}$ & $79.1 \pm 1.4$ & $8.3 \pm 0.3$ \\
\hline S13 & $96.1 \pm 0.6$ & $7.3 \pm 0.3$ & NOA 73 & Wrinkling & $\begin{array}{l}20 \%, \\
300 \mathrm{~min} \\
\end{array}$ & $95.7 \pm 0.7$ & $6.8 \pm 0.7$ \\
\hline S14 & $96.2 \pm 1.3$ & $6.4 \pm 0.7$ & NOA 81 & Wrinkling & $\begin{array}{l}20 \%, \\
300 \text { min }\end{array}$ & $96.7 \pm 0.1$ & $6.2 \pm 1.0$ \\
\hline S15 & n.a. ${ }^{*}$ & n.a.* & NOA 73 & 3D-printing & - & $130 \pm 15.6$ & $46.6 \pm 5.4$ \\
\hline S16 & $118 \pm 17$ & $47 \pm 6$ & NOA 81 & 3D-printing & - & $112 \pm 6.2$ & $47.0 \pm 2.6$ \\
\hline
\end{tabular}

*Faulty measurements discovered after the perception experiment, thus not possible to redo as a before measurements. 


\subsection{Tactile friction measurements}

Tactile friction measurements were performed using a ForceBoard ${ }^{\circledR}$ (Industrial Dynamics Sweden AB, Järfälla, Sweden), equipped with both horizontal and tangential load cells, consisting of strain gauges in a Wheatstone bridge configuration. A mechanical loading results in proportional voltage changes. Forces were sampled at $100 \mathrm{~Hz}$ using DAQFactory software. The friction force $(F)$ and applied load $(L)$ were continuously recorded as a finger interrogated the model skin surface by moving the index finger back and forth, and the friction coefficients $(\mu)$ were calculated using Amontons friction law as the ratio of $F$ to $L$. The model surface samples were mounted on the top plate of the force sensor with double sided adhesive tape.

Friction was recorded in two ways; 1) by using the same finger under controlled conditions and 2) by five of the participants during the slipperiness assessment in the perception study. In the first case, friction between the human finger (dominant index finger) of one single experimenter and the various model surfaces was measured in controlled environment $\left(\mathrm{T}=23+/-1^{\circ} \mathrm{C}\right.$ and $\left.\mathrm{RH}=50+/-2 \%\right)$. Six measurements were performed for each of the 16 samples in random order, i.e. in total 96 measurements. For each friction experiment the index finger, inclined at about $30^{\circ}$, was stroked forward (away from the body) and back (towards the body) 15 times over the sample area as described previously [8]. Both the average friction coefficient of the whole measurement (COF 15) and for the first three stroking cycles (COF 3) was calculated. The average stroking distance was about $40 \mathrm{~mm}$ for surfaces S1-S14 and $30 \mathrm{~mm}$ for surfaces S15-S16 due to the different dimensions of the latter substrates. The average speed of all measurements was $40 \mathrm{~mm} / \mathrm{s}$ and the average applied load for all measurements was $1.1 \pm 0.2 \mathrm{~N}$. The moisture content of the finger was measured using a Corneometer (CM825, Courage Khazaka Electronic $\mathrm{GmbH}$ ) prior to each test. Five individual moisture content measurements were performed for each moisture content analysis. The finger was cleaned with neutral soap, rinsed thoroughly with water to remove soap residues, dried with a clean towel and then allowed an equilibrium period of approximately 5 minutes prior to each measurement session. In the case of the five participants (P9-P13), friction was measured between the human finger (dominant index finger) and the various model surfaces during slipperiness assessment as a part of the perception session ( $16 \times 6$ measurements). The load, number of stroking cycles, stroking distance, speed and inclination of the finger were all uncontrolled for all participants and they were not given any specific instructions, except that of a minimum stroking time of approximately three seconds. The room temperature and humidity was noted during each experiment. The moisture content of the finger was measured before initiating the experiment, after finalizing the experiment and at two time points during the experiment. The finger was cleaned approximately 10 minutes before initiating the study.

\subsection{Contact area estimation}


The real area of contact between a human finger and the different structured surfaces was estimated with the method used by Duvefelt et al. [51] for similar surfaces. In this model, the surfaces of the fingerprint ridges are considered flat and relatively large compared to the surface features. The measured wavelengths and amplitudes of the surface structures were used as input in the model which assumed a 1D sine function superimposed on a plane [52]. Figure 2 shows the skin (dotted line) deformed by a distance $\delta$ against the test surface (black sinusoidal) resulting in contacts of width $2 a$.

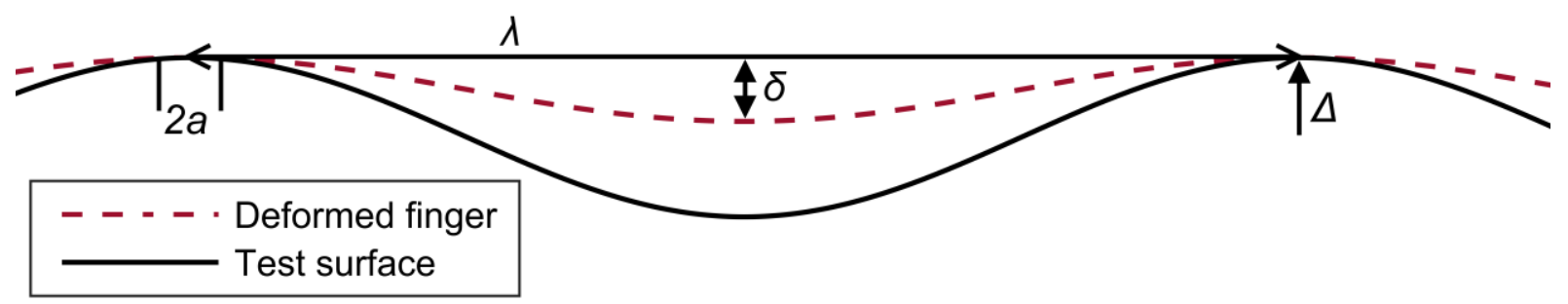

Figure 2. Model of contact between flat skin and the test surface. Amplitude of the test surface $\Delta$ and wavelength $\lambda$ are known while deformation depth $\delta$ and contact width $2 a$ were calculated.

The contact width $2 a$ is calculated by:

$$
2 a=\frac{2 \lambda}{\pi} \sin ^{-1}\left(\frac{\bar{p}}{p^{*}}\right)^{1 / 2}
$$

where $\bar{p}$ is the mean surface pressure obtained from the ratio of normal force and apparent contact area and $p^{*}$ is the pressure needed for full continuous contact given by:

$$
p^{*}=\pi E^{*} \frac{A}{\lambda}
$$

The combined Young's modulus $E^{*}$ is calculated from the Young's modulus's and Poisson's ratios for the two surfaces. The values for the two different NOAs were supplied by the manufacturer and values for the skin were taken from the literature $[53,54]$. The values of the variables used in the calculation of contact width are shown in Table 2. 
Table 2. Used properties for the skin, test surface, apparent contact area and normal force used when calculating the contact width.

\begin{tabular}{|l|l|}
\hline Young's modulus for finger, $E_{f}[53]$ & Variable \\
\hline Poisson's ratio for finger, $v_{f}[54]$ & 0.4 \\
\hline Young's modulus for NOA73, $E_{\text {NOA73 }}$ & $11 \mathrm{MPa}$ \\
\hline Young's modulus for NOA81, $E_{\text {NOA81 }}$ & $1379 \mathrm{MPa}$ \\
\hline Poisson's ratio for test surfaces, $v_{N O A}$ & 0.4 \\
\hline Combined Young's modulus, $E^{* 73}$ & Variable \\
\hline Combined Young's modulus, $E^{* 81}$ & Variable \\
\hline Area encompassed by model & $113 \mathrm{~mm}^{2}$ \\
\hline Normal force & $1 \mathrm{~N}$ \\
\hline
\end{tabular}

A simple model for the fingerprint geometry was used to calculate the total contact length. A circular fingerprint with a diameter of $12 \mathrm{~mm}$ was used. Each ridgetop and gap were set to $250 \mu \mathrm{m}$, estimated from a microstructural cross-section of a middle finger using optical coherence tomography [18]. This gives an apparent contact area against a perfectly smooth surface of $56 \mathrm{~mm}^{2}$ and a total of 12 ridges, see Figure 3.

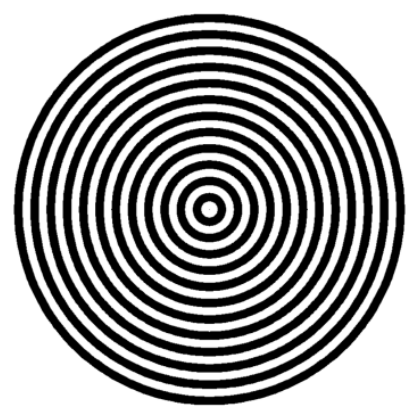

Figure 3. Schematic of the fingerprint used in model. The diameter is $12 \mathrm{~mm}$, the ridge width and gap are both $250 \mu \mathrm{m}$.

Placing the sinusoidal test surface on this fingerprint pattern would result in a series of contact lines intersecting the (black) ridges in Figure 3. The separation between the lines is given by the wavelength of the test surface, and a shorter wavelength thus leads to more lines. The width of the lines depends on both the wavelength and amplitude. For example large wavelengths and low amplitudes lead to lower curvature and thus a broader line contact against the finger, whereas a large amplitude increases the curvature and narrows the line. The total contact area is obtained by summing the area of the contact lines projected onto the ridge pattern in Figure 3. The model is highly simplified, and ignores the topographical variation in the fingerprint ridges. This simplification is expected to be reasonable if the 
modulus of the finger is less than that of the surface, if the deformation is comparable to the skin's local topography (due to conformability) and when the deformation is smaller than the amplitude of the ridges. All these criteria would appear to be met in this case, however in a worst case, or if these criteria were not to be met, the model would still supply a useful, systematic scaling of the true area of contact.

\subsection{Psychophysical study}

The psychophysical study is aimed at measuring perceived slipperiness and perceived roughness of fine textures (below $200 \mu \mathrm{m}$ ) and to link the perceptions with surface properties. The materials are relatively rigid surfaces (in comparison to the finger) with no immediately apparent, discernible difference in compliance. Since a previous study on a similar set of materials yielded a 2-dimensional space, perceived softness was not evaluated as a parameter on its own but rather the hardness of the photopolymer was considered a factor to mediate perceived roughness and slipperiness. Sixteen participants scaled a total of 16 stimuli (8 different wave patterns on 2 different materials, Table 1) using the method of free magnitude estimation [55] with 6 repetitions of each stimulus, i.e. $96(6 * 16)$ judgments in total for each perception and person. Each participant was assigned a unique randomized presentation order. The randomization was made pairwise with the result that during the experiment, each of the 8 different patterns were paired up with the same pattern, but on the other material. This pairwise randomization was made to maximize the chance to detect material differences between the NOA73 and NOA 81 samples. Half of the participants got a pairing order with NOA 73 first and the other half with NOA81 first. To further explore the contribution of friction, a subset of the participants conducted friction measurements simultaneously with the slipperiness measurements. The purpose of this addition was twofold, firstly to increase the ecological validity of the friction measurements i.e. to measure the friction coefficient in a more natural handling situation, and also to examine the representativeness of the experimental finger used in the more controlled friction measurement.

\subsubsection{Participants}

For the perception experiment 16 women (ages 20-33, average 24.4) were recruited. All participants were right handed. 12 participants were students at KTH Royal Institute of Technology and 4 were SP (Technical Research Institute of Sweden) employees with no connection to the current study. All participants received two theatre tickets as compensation for completing the experiment.

\subsubsection{Perception experiment procedure}


Before the experiment started, the participants were asked to wash their hands with soap and water. The participants then received written instructions on how to complete the experiment, as well as a common definition of roughness and slipperiness; in both English and Swedish. The haptic instruction was to use the index finger of their dominant hand (the right hand in all 16 cases) and stroke the surface back and forth over the ridges until they were ready to give an estimate. After the instructions were read and any questions clarified, the participants were blindfolded. A short practice session preceded the experiment to give the participants a gist of the range of materials and to practice the scaling procedure. Between the two sessions there was a short break in which the participants washed their hands again. The participants were free to complete the experiment at their own pace, and were usually completed in approximately one and a half hours. Five of the participants (9-13) also conducted friction measurements while scaling slipperiness; the details on this procedure are described in 2.2 .

Free magnitude estimation is a well-used method to measure perceptual quantities [56]. The principle behind magnitude estimation is to ask the participants to assign a number that corresponds to the perceived intensity of the stimulus. When the next stimulus is presented the participant is instructed to assign a number that is proportionate to the previous judgment. For example, if the first stimulus is perceived as 10 and the next stimulus is twice as intense it will be a 20. Each stimulus is then repeated a number of times to acquire an average intensity for the stimulus. To lessen the impact of "off judgments" it is customary to calculate the geometric mean rather than the arithmetic mean. The "free" in free magnitude estimation refers to the freedom of the participant to use her own reference modulus rather than having the experimenter decide the reference modulus.

\section{Results and Discussion}

\subsection{Tactile friction}

Tactile friction coefficients for the different surfaces determined by a single experimenter at controlled ambient conditions, calculated from the first three stroking cycles (COF3), are presented in Figure 4A. The

first three stroking cycles are used because this more closely mimics the action of touching and assessing a material. Fifteen stroking cycles have been used as a control for check for variations in friction over time. In previous studies where the friction has been measured by participants during perception assessment, a change in friction coefficient was observed as a result of altering the applied load in response to the friction experienced during the initial stroking cycles (to obtain a more pleasant contact) [37].

The results in Figure 4A show a dramatic decrease in friction of the material when structure is introduced, compared to the reference materials with no systematic pattern $(\lambda=0 \mu \mathrm{m})$. The friction coefficients are similar for all materials with wavelengths in the region 30-80 and $120 \mu \mathrm{m}$, whereas an increase of the friction coefficient is observed around $100 \mu \mathrm{m}$. Since skin hydration is considered an important factor of the friction response $[20,22,24,25,57]$, the skin hydration was measured prior to each friction measurement. 

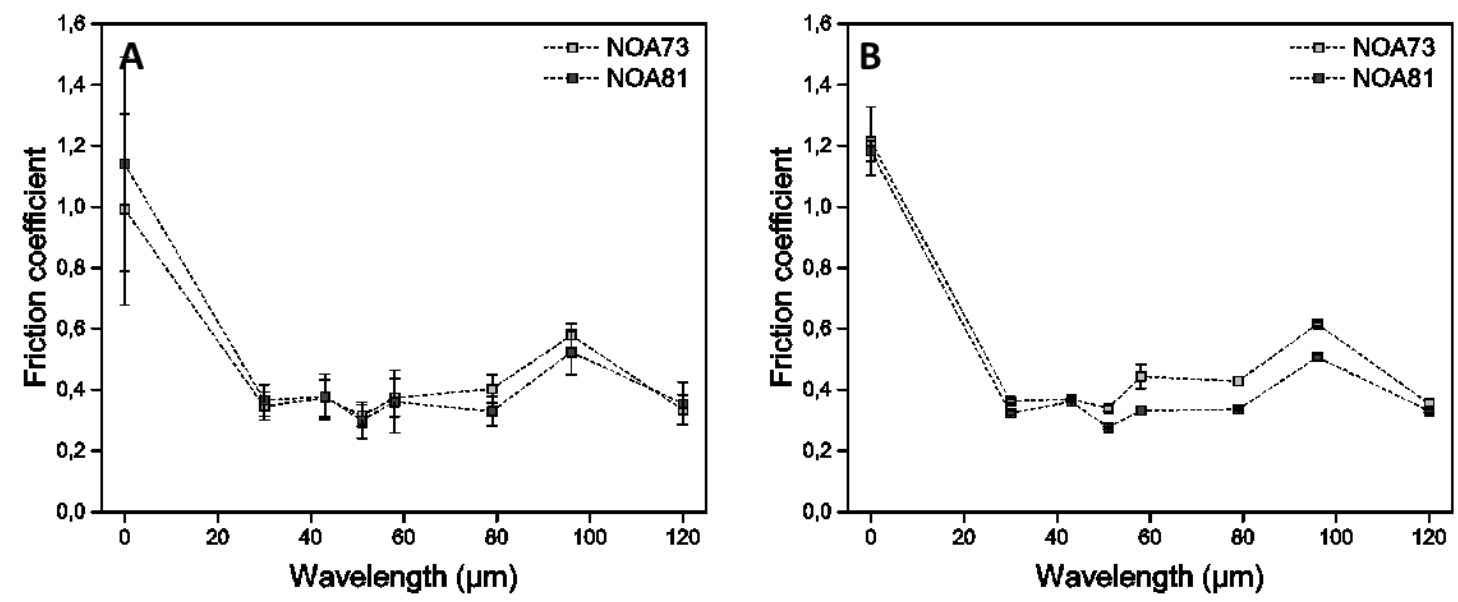

Figure 4. Friction coefficient versus wrinkle wavelength for the 16 surfaces obtained with one experimental finger presented as average and standard deviation of 6 measurements $(A)$ and as Friction coefficients normalized with skin hydration (B).

\subsubsection{Tactile friction and skin hydration}

Skin moisture/hydration was measured by electrical capacitance by means a Corneometer ${ }^{\circledR}$ CM 825 (Courage \& Khazaka Electronic $\mathrm{GmbH}$, Cologne, Germany). The method is based on capacitance, and that water has a high dielectric constant (81) compared to the other substances on the finger. The skin hydration is expressed in arbitrary units (a.u.) between 0 and 130. For healthy skin and normal room conditions ( $20^{\circ} \mathrm{C}$ and $40-60 \%$ relative humidity), the manufacturer's experience and interpretation of the moisture values are for very dry skin (<30), dry skin (30-40) and sufficiently moist $(>40)$. The measured skin hydration at the fingertip varied between 29-52 a.u. for the same finger (single experimenter) during the measurements (average value $44 \pm 6$ ). The observed general trend that higher hydration values gave rise to higher friction coefficients for the same material are shown for two surfaces in Figure 5 . This effect of moisture is in agreement with what has been previously reported e.g. [17, 58, 59]. Although the effect of moisture is well documented, we are not aware of any efforts to normalize or scale tactile friction with moisture. Thus, we suggest a method to normalize the friction coefficient with the moisture level in the finger for repeated measurements with the same experimental finger. This method could also be used in studies with different persons with even greater individual variations in finger hydration to compare friction coefficients within a set of surfaces. For each surface, the calculated friction coefficient for each measurement was plotted versus the skin hydration obtained immediately prior to that specific measurement. A straight line was fitted to the six data points as exemplified in Figure 5. The normalized friction coefficient for each surface was then obtained from the fitted equation for $x=45$, i.e. the calculated friction coefficient according to the fitted linear equation where the skin hydration is 45 a.u. $\left(\right.$ COF $_{\text {corr }}=$ slope* $45+$ intercept). The hydration value of 45 is around the average hydration detected for the finger used in the study. The error of these normalized friction coefficients are taken as the standard deviation but calculated with the degrees of freedom set to $n-2$ since both the slope and intercept of the fitted line are used for the normalization. The normalized friction coefficients are displayed in Figure 4B. 
The linear fit approach for normalization with skin hydration gives similar mean values as the mean of all measurements before normalization. However, the deviation between experiments is smaller. In the present work only one experimenter performed the measurements and these were carried out under controlled relative humidity and temperature, but the measurements were nonetheless influenced by that person's skin hydration level. The approach would probably be even more useful to normalize the tactile friction coefficients obtained with different persons. The skin moisture was measured prior to each series of friction measurements for the five participants.

As can be seen, the differences in friction coefficients between the two materials are significant, showing higher values for the more compliant material NOA73 (lower elastic modulus) compared to NOA81 (Figure $4 \mathrm{~B})$. There are only marginal differences in the chemistry of the resins, so the difference in friction coefficients almost certainly reflects the elastic modulus difference. This indicates that surfaces of differing elastic modulus, at least over the range spanned here, can be distinguished via their frictional interactions of the human finger. The reasons for this are discussed below in the context of the surface area.
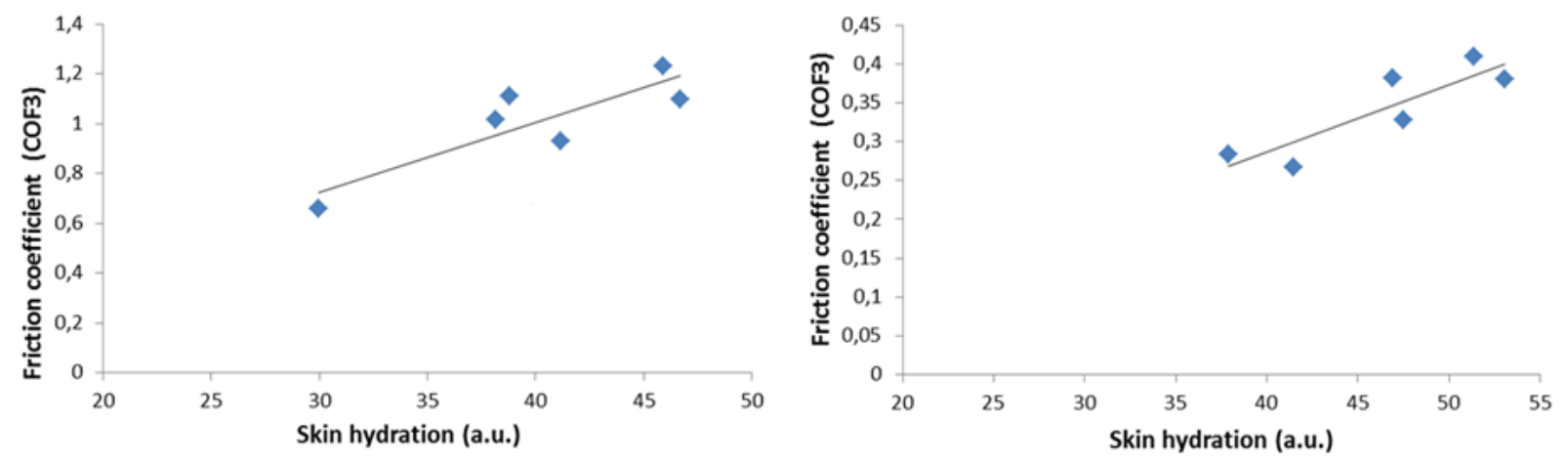

Figure 5. Relation between skin hydration and friction coefficient for a single individual on two of the surfaces included in the study, S1 (left) and S16 (right). The friction coefficient can be defined by a least squares linear fit to the data and taking the value at any arbitrary value (in our case 45, which is the average hydration level detected in this study)

The friction measurements with the five participants had lower experimental control since the participants had greater inter-individual variations in skin hydration, they applied their own preferred load and speed, and consequently there were differences in the absolute values of the friction coefficients. However, the trends observed are similar for all participants, where the friction coefficients for the reference materials are dramatically higher compared to the other surfaces, the friction coefficients for the materials with wavelengths $30-80$ and $120 \mu \mathrm{m}$ are in the same range, while a small increase in the friction coefficient was observed for materials with wavelengths 80-100 $\mu \mathrm{m}$. In order to be able to compare the data recorded in controlled conditions and by the five participants, the scales were re-defined based on the assumption that the individual coefficients are dependent on the individual variations across the fingers but are proportionally consistent within each finger, and can thus be equated to individual use of scales, much like in free magnitude estimation [56]. Thus, the scales were re-defined so that each individual finger was 
assumed to generate proportionally equivalent responses across the surfaces, but with differences in the magnitude of the response. Based on this assumption, the ratio between the average total response and the average individual response for each individual was calculated. Each data set was then multiplied with the corresponding ratio, whereupon the average normalized friction coefficients measured by the five participants from the perception study were compared to the normalized friction coefficients measured under controlled conditions as shown in Figure 6. It is clear that the trends are very similar for the group who performed the tactile friction measurements during slipperiness assessment and the one experimenter performing the measurements in controlled conditions, showing that it is possible to obtain general trends in variation of tactile friction properties by using the finger of a single experimenter for the measurements, when comparing a group of samples.

Where the skin hydration has been studied, and is related to stick-slip behaviour, in relation to grip and object manipulation e.g [59] and for textiles [17] it is has not been studied in hard textured surfaces or in active exploration tasks. Given the demonstrated effects of moisture on grip and friction, where there is no apparent texture on the surface, a model to normalize the friction coefficient with skin hydration would be useful for establishing the relative importance of roughness and slipperiness and their psychophysical relation to the surface. The normalization methods proposed here provide a means to compare "noisy" individual friction measurements. The similarity of the two normalization results indicate that a trained experimenter with moisture normalization will capture the friction trends for a group of participants.

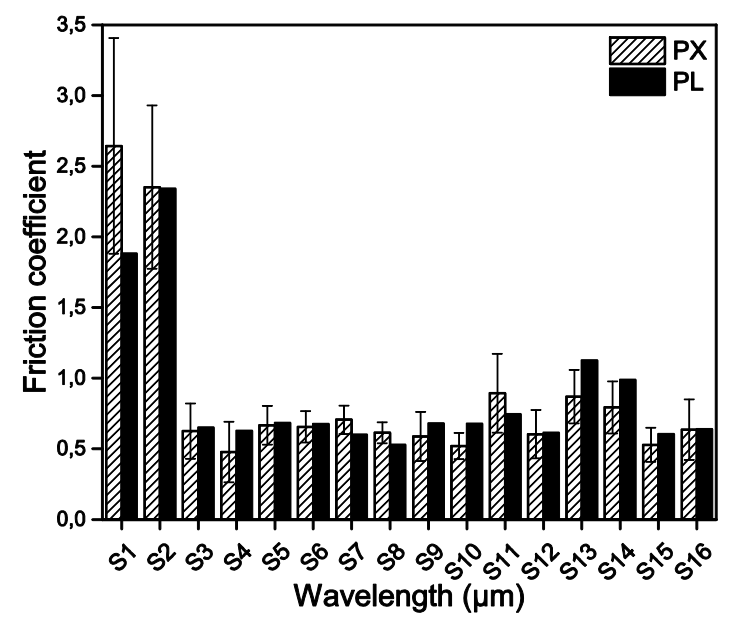

Figure 6. Average friction coefficient normalized over the five participants during slipperiness assessment (striped bars) and normalized friction coefficients measured by one experimenter in controlled conditions (black bars).

\subsection{Contact area}

To explain the differences in friction coefficient it is necessary to address the issue of the true area of contact between the finger and the surface in question. Earlier results show that there is a linear relationship between friction and load in the load range used $(\geq 1 N)$. This relationship has been shown to 
be non-linear and thus reflect finger pad deformation at lower loads. At higher loads however it can be assumed that the projected contact area does not change, and the Amontonsian behaviour is attributable to adhesive friction and local changes. This estimated real contact area, obtained using equation 2, is plotted versus the wrinkle wavelength. The non-monotonic dependence of the friction coefficient on wavelength which is visible in Figure 4 is largely reproduced by the contact area calculations. Thus, the friction "peak" observed at $100 \mu \mathrm{m}$ is most probably due to a higher real contact area at the finger-surface interface. These results indicate that the main friction mechanism in tactile exploration of fine textures is adhesive friction [19].

The fact that there is a systematic difference in the measured friction coefficients between the two polymer materials indicates that there are differences in the contact area against the two polymers. While such differences are seen in Figure 7 the differences are not as pronounced as those of the friction coefficients in Figure 4. Given that the moduli of the polymers are both considerably larger than that of the 0.2MPa used by Duvefelt et al. [51], it is somewhat surprising that the friction differences should be so large. This observation implies that the skin modulus should be closer in magnitude to that of the polymers. In the supplementary information we show calculated contact areas for the two polymers against the model fingertip as a function of the fingertip elastic modulus for all the topographies used. By comparing the relative values for the two polymers at the different wavelengths with relative magnitudes of the friction data (assuming that there is a direct correlation) then it can be estimated that the value of the elastic modulus which best fits the observations is of the order of $10 \mathrm{MPa}$ (though the simplicity of the model means that this is a rather crude estimate) If it is considered that the deformation leading to the contact area is small and local, it might make more sense to use the elastic modulus of the stratum corneum. AFM penetration experiments on human stratum corneum using a single hair fibre have recently been published, and suggest that the modulus varies from $2 \mathrm{MPa}$ to around $430 \mathrm{MPa}$ depending on the dimensions of the deformation [60]. The inferred value of ca $10 \mathrm{MPa}$ from considering contact areas and friction is in good agreement with the lower values in this range which correspond to deformations of the same order as in this study. This indirectly provides support for the physical usefulness of the model since its combination with the friction data actually allows the skin's elastic modulus to be inferred. 


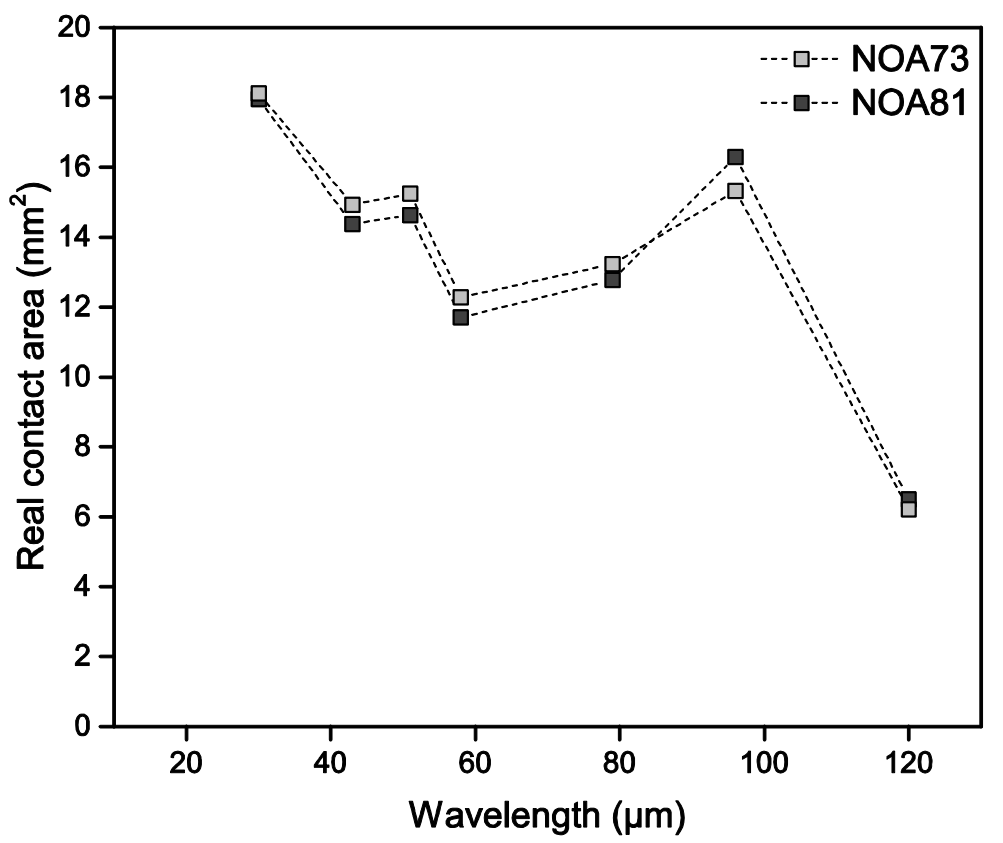

Figure 7. The estimated real contact area versus wrinkle wavelength assuming the low value of $0.2 \mathrm{MPa}$ for the Young's modulus of the finger as done in[51]. A more reasonable value is inferred to be ca $10 \mathrm{MPa}$. The estimated value of a human finger versus a completely smooth surface is $56 \mathrm{~mm}^{2}$.

\subsection{Tactile perception of roughness and slipperiness}

The results of the perception study show a distinct differentiation between the unwrinkled and the wrinkled surfaces (Figure 8) with clear wavelength dependence for both perceived roughness and perceived slipperiness. Over the $30 \mu \mathrm{m}$ to $52 \mu \mathrm{m}$ wavelength span there was no change in perceived slipperiness and from the $58 \mu \mathrm{m}$ surfaces and up to $120 \mu \mathrm{m}$ there was a steady decline in perceived slipperiness. For perceived roughness there was no big change in the range between $30 \mu \mathrm{m}$ to $80 \mu \mathrm{m}$ wavelengths, but at $100 \mu \mathrm{m}$ and even more clearly at $120 \mu \mathrm{m}$, perceived roughness starts to become significant.

A clear separation between blank/unstructured reference materials and all patterned surfaces was detected, in terms of friction, slipperiness perception and roughness perception. However, the high friction blank surfaces were perceived as rough. The similar roughness judgments between the blank surfaces and the more coarsely patterned surfaces is also in clear contrast to the similarities observed in the previous study on wrinkled model surfaces [10] where the two types of surfaces are perceived as very different; this tells us that there is more to roughness and slipperiness perception than what is captured in the measurements. There are two possible explanations for this. One explanation is that there is a 
dependency between perceived slipperiness and roughness, which might also tie into the earlier mentioned difficulty to find a reliable third dimension of textures. Another explanation is that there are two different kinds of roughness. It was proposed by Bergmann Tiest and Kappers [34] that roughness might be a multidimensional perception in itself. If this is the case, the observed elevation in perceived roughness indicates that people rely on both friction induced roughness detection and topographically induced roughness detection.

A main finding is the $100 \mu \mathrm{m}$ range which seems to be a breaking point for perceived roughness. Below $100 \mu \mathrm{m}$ the changes in perception observed by Skedung, et al. [10] seem to be dominated by slipperiness and best represented by the friction coefficient. As the texture increases above $100 \mu \mathrm{m}$, roughness starts to become a more discernible perception.
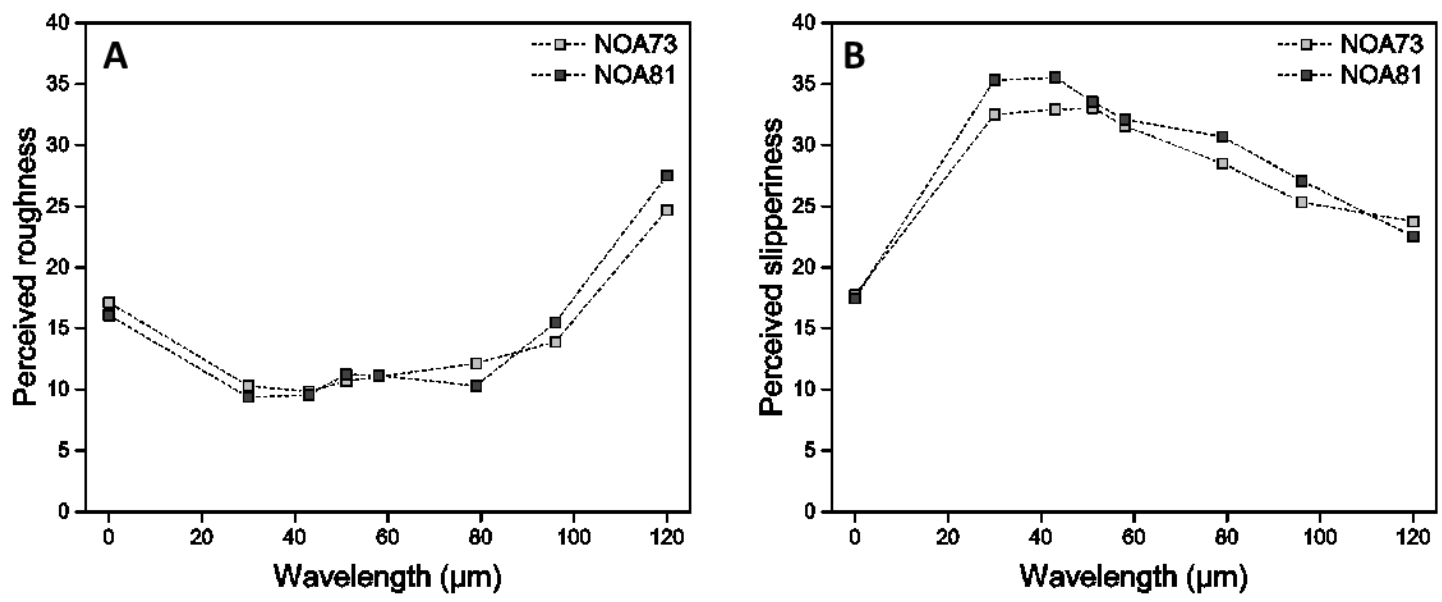

Figure 8. Perceived roughness (left) and perceived slipperiness (right) plotted against the wavelength.

As shown in Figure 9, there are two sets of surfaces that fall out on the friction axis in the comparison of perceived slipperiness with friction coefficient. These are the unwrinkled surfaces and the $96 \mu \mathrm{m}$ surfaces that also display the friction maxima observed in Figure 4, Figure 5 and Figure 7. The $120 \mu \mathrm{m}$ surfaces are perceived as less slippery than the big cluster, but do not have a significantly lower friction coefficient than the clustered values. This suggests that the $120 \mu \mathrm{m}$ surfaces are perceived as less slippery on the basis of the spatial period of the pattern and not the friction coefficient. The reason for this might be the increased presence of perceived roughness that reduces the importance of perceived slipperiness. The surfaces in the $30 \mu \mathrm{m}-52 \mu \mathrm{m}$ range can be described as smooth and slippery, and beyond this interval an increased wavelength will reduce slipperiness and in time (at $\lambda>100 \mu \mathrm{m})$ increase roughness. 

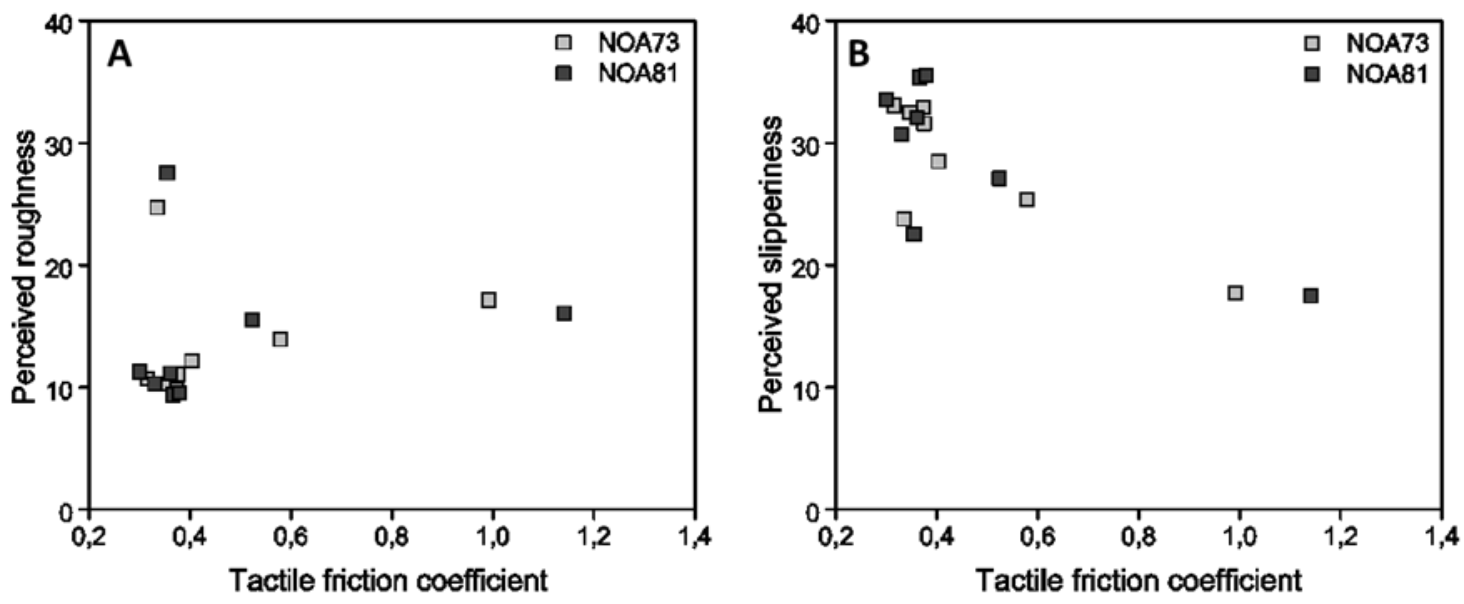

Figure 9. Perceived roughness and perceived slipperiness plotted against the high control condition tactile friction coefficient.

The friction and perceptual peak at $96 \mu \mathrm{m}$ can also be seen in the individual data for the participants. For visualization purposes we inverted the slipperiness scores for participant 12 and there was a striking resemblance between the perception and friction plots, Figure 10. The friction spike between 80 and $100 \mu \mathrm{m}$ matches the observed perceptual change for participant 12 . The close resemblance from both the perception data and the friction data to the data obtained in [10] both validate those findings and point to slipperiness as being the main factor in that study.

A difference between the two different materials (which vary in elasticity) was also observed in the perception study. The perceived slipperiness of the softer material was systematically lower and this correlates extremely well with the observation that the friction coefficient was higher. This once again indicates that friction is a sensitive measure of perception and we are able to detect differences in moduli that are large compared to what has traditionally been thought of as the finger modulus. It is therefore highly likely that larger differences would be detectable for more flexible materials and this is the subject of a future study in our laboratories. 

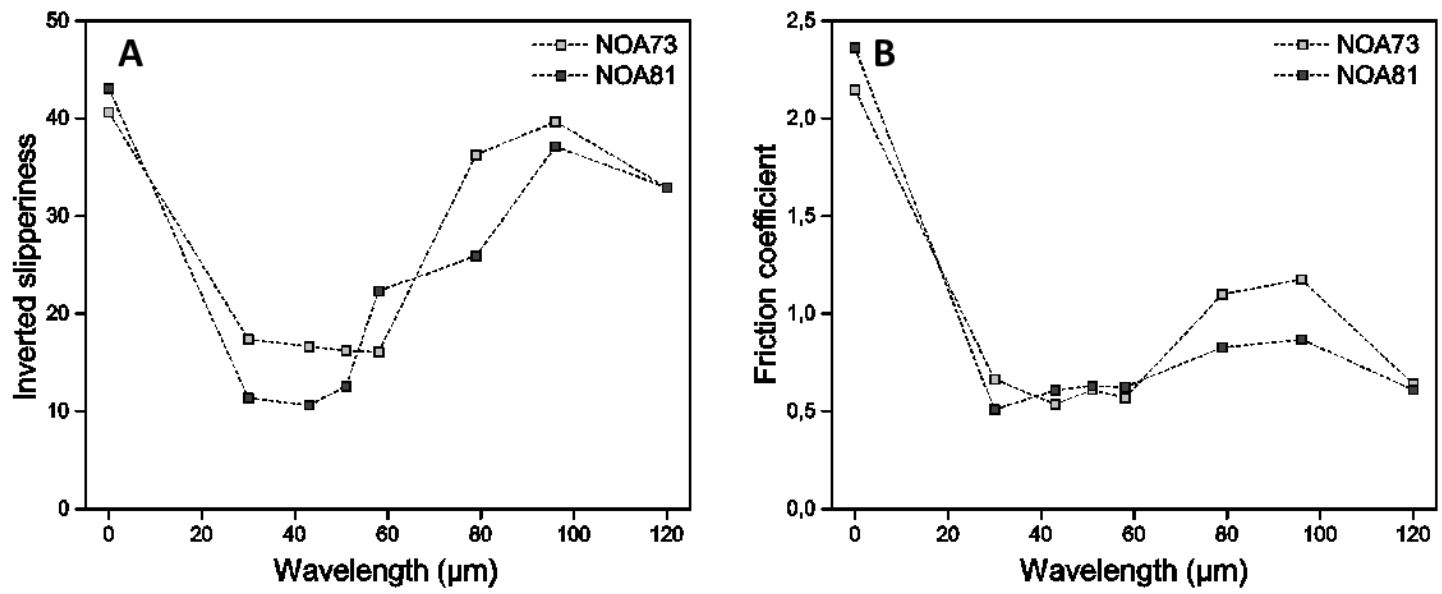

Figure 10. A, the inverted slipperiness for participant 12 plotted against the wavelength. B, the friction coefficient for participant 12 plotted against the wavelength.

\subsubsection{Data quality for the perception measurements}

Several actions were taken to ensure a high data quality in the perception experiments. The experimental design included a test for order effects. The presentation of surface structures was randomized for each participant, but the NOA order was manipulated so that half of the participants received NOA73 first followed by the same structure but with NOA81, and the other half of the participants received NOA81 first. The presentation order did not seem to affect the outcome of the experiment. The order of the sessions was also split in half, so that half of the participants scaled roughness first and half of the participants scaled slipperiness first. This was done to observe if there was any learning or fatigue effects while completing the experiment. The results indicate that there were no learning or fatigue effects. There were, of course, individual differences amongst the 16 participants. Some managed the task better than others, but the majority of the participants exhibited a pattern that resembles the average well. An example of an individual plot compared to the overall plot is shown in the supplementary information. 


\section{Conclusions}

The results cement the increasing body of knowledge concerning the role of tactile friction and the associated perception of slipperiness as key aspects of fine texture perception. While earlier focus has been very much on topography, for which "roughness" might be considered the appropriate perceptual attribute, it becomes clear from this work that "slipperiness" is a more nuanced attribute for spatial periods below $100 \mu \mathrm{m}$. Interestingly, very smooth surfaces are anomalously perceived as rougher than textured surfaces, due to the very high resistance to sliding encountered as a result of the intimate mating of the skin with the surface, and possibly due to the associated tendency for increased large scale stickslip.

In terms of reliably interpreting trends and connecting tactile friction to perception, both group data and data from a trained individual can be used; the latter approach provides more sensitive correlations and thus predictability. One of the reasons for this is the fact that differences in friction forces measured between individuals is typically of similar order or sometimes greater than, the differences measured between the different surfaces. (The trends are nonetheless consistent.) One of the main reasons for this variation, and indeed for the variation in results obtained from a single individual, is variation in finger moisture [8]. Thonnard, Derler [19, 58, 59] and others have also shown that moisture is an important consideration in tactile friction, and this paper quantitatively emphasis that this effect is very important for non-adsorbing surfaces. (Earlier studies comparing individual to single experimenter friction were performed on more adsorbent paper surfaces.) A further advance of this work is to show how the reliability of the correlations can be increased by correcting the friction data for moisture, and the presentation of a simple method to do so.

The trends can all be explained in terms of a simple contact mechanical model which takes into account relative changes in the real area of contact. (The absolute value of the contact area should strictly include the surface roughness of the skin, but this level of sophistication appears unnecessary for systematic relative evaluations, possibly due to its lower modulus than the surfaces employed in this case). Use of the model reveals that the relevant modulus for use in the contact mechanical approach is much higher than ordinarily invoked, and reflects the modulus of the stratum corneum rather than that of tissue. This is in line with the scale of the deformations, expected, and the fact that at the loads used to probe surfaces and measure tactile friction (of the order of a Newton or so) the fleshy deformation is largely saturated such that the friction-Load regime follows a pseudo Amontonsian behaviour.

Finally, this deformation model allows us to explain the fact that two resins of different elastic modulus, but identical chemistry and topography can be systematically discriminated on the basis of measured tactile friction, and perceived slipperiness. To our knowledge this is the first time it has been possible to isolate the elastic modulus from other parameters in this fashion.

A recent study looked at tactile discrimination of textureless surfaces of different materials[61]. It clarifies that, at least in the case of smooth surfaces, their discrimination is dependent on the onset of slip. Thus chemistry, elastic modulus, humidity and $\mu \mathrm{m}$ scale topography are all important in perception. All these 
factors contribute in a quantifiable manner to the tactile friction, which is thus demonstrably the key parameter for understanding and prediction of fine texture tactile perception .

\section{Ethical Approval}

All procedures performed in studies involving human participants were in accordance with the ethical standards of the institutional and/or national research committee and with the 1964 Helsinki declaration and its later amendments or comparable ethical standards.

Informed consent was obtained from all individual participants included in the study. None of the experiments were invasive or harmful physiologically or psychologically. All data was stored anonymously, participations was voluntary and the participants had the right to quit at any time if they so wished. 


\section{Acknowledgements}

The authors would like to acknowledge Johan Andersson, Karin Hallstensson and Mimmi Eriksson for expert technical support and aid with data collection. Vicky Polashock and Keyur Desai are gratefully acknowledged for valuable discussions. Anders Söderberg is acknowledged for help with MATLAB programming.

\section{Declaration of interests}

Conflicts of interest: none.

\section{Role of the funding source}

This research did not receive any specific grant from funding agencies in the public, commercial, or notfor-profit sectors.

\section{References}

1. Childs, T. and B. Henson, Human tactile perception of screen-printed surfaces: Self-report and contact mechanics experiments. Proceedings of the Institution of Mechanical Engineers, Part J: Journal of Engineering Tribology, 2007. 221(3): p. 427-441.

2. Darden, M.A. and C.J. Schwartz, Investigation of skin tribology and its effects on the tactile attributes of polymer fabrics. Wear, 2009. 267(5): p. 1289-1294.

3. van Kuilenburg, J., M.A. Masen, and E. van der Heide, A review of fingerpad contact mechanics and friction and how this affects tactile perception. Proceedings of the Institution of Mechanical Engineers, Part J: Journal of Engineering Tribology, 2015. 229(3): p. 243-258.

4. Van Kuilenburg, J., et al., An experimental study on the relation between surface texture and tactile friction. Tribology international, 2012. 48: p. 15-21.

5. Dinç, O., et al., Some parameters affecting tactile friction. Journal of tribology, 1991. 113(3): $\mathrm{p}$. 512-517.

6. Gee, M., et al., A new friction measurement system for the frictional component of touch. Wear, 2005. 259(7): p. 1437-1442.

7. Derler, S., et al., Friction of human skin against smooth and rough glass as a function of the contact pressure. Tribology International, 2009. 42(11): p. 1565-1574.

8. Skedung, L., et al., Finger friction measurements on coated and uncoated printing papers. Tribology letters, 2010. 37(2): p. 389-399.

9. Skedung, L., et al., Tactile perception: finger friction, surface roughness and perceived coarseness. Tribology International, 2011. 44(5): p. 505-512.

10. Skedung, L., et al., Feeling Small: Exploring the Tactile Perception Limits. Sci. Rep., 2013. 3.

11. Smith, A.M., et al., Role of friction and tangential force variation in the subjective scaling of tactile roughness. Experimental Brain Research, 2002. 144(2): p. 211-223.

12. Masen, M., A systems based experimental approach to tactile friction. Journal of the mechanical behavior of biomedical materials, 2011. 4(8): p. 1620-1626.

13. Derler, S., et al., Influence of variations in the pressure distribution on the friction of the finger pad. Tribology International, 2013. 63: p. 14-20.

14. Derler, S., et al., Friction mechanisms and abrasion of the human finger pad in contact with rough surfaces. Tribology International, 2015. 
15. Derler, S., R. Rossi, and G.-M. Rotaru, Understanding the variation of friction coefficients of human skin as a function of skin hydration and interfacial water films. Proceedings of the Institution of Mechanical Engineers, Part J: Journal of Engineering Tribology, 2015. 229(3): p. 285-293.

16. Derler, S., U. Schrade, and L.-C. Gerhardt, Tribology of human skin and mechanical skin equivalents in contact with textiles. Wear, 2007. 263: p. 1112-1116.

17. Gerhardt, L.C., et al., Influence of epidermal hydration on the friction of human skin against textiles. Journal Of The Royal Society Interface

2008. 5(28): p. 1317-1328.

18. Adams, M.J., et al., Finger pad friction and its role in grip and touch. Journal of The Royal Society Interface, 2013. 10(80): p. 20120467.

19. Derler, S. and L.-C. Gerhardt, Tribology of skin: review and analysis of experimental results for the friction coefficient of human skin. Tribology Letters, 2012. 45(1): p. 1-27.

20. Pasumarty, S.M., et al., Friction of the human finger pad: influence of moisture, occlusion and velocity. Tribology Letters, 2011. 44(2): p. 117-137.

21. Tomlinson, S.E., et al., Understanding the Friction Mechanisms Between the Human Finger and Flat Contacting Surfaces in Moist Conditions. Tribology Letters, 2011. 41(1): p. 283-294.

22. Tomlinson, S.E., R. Lewis, and M.J. Carre, The effect of normal force and roughness on friction in human finger contact. Wear, 2009. 267: p. 1311-1318.

23. Tomlinson, S.E., et al., Human finger contact with small, triangular ridged surfaces. Wear, 2011. 271(9-10): p. 2346-2353.

24. Buchholz, B., L.J. Frederick, and T.J. Armstrong, An investigation of human palmar skin friction and the effects of materials, pinch force and moisture Ergonomics, 1988. 31(3): p. 317-325.

25. Andre, T., P. Lefevre, and J.L. Thonnard, A continuous measure of fingertip friction during precision grip. Journal Of Neuroscience Methods, 2009. 179(2): p. 224-229.

26. Liu, X., et al., Quantifying touch-feel perception: tribological aspects. Measurement Science and Technology, 2008. 19(8): p. 084007.

27. Barnes, C., et al., Surface finish and touch-a case study in a new human factors tribology. Wear, 2004. 257(7): p. 740-750.

28. Chen, X., et al., Exploring Relationships between Touch Perception and Surface Physical Properties. International Journal of Design, 2009. 3(2): p. 67-76.

29. Chen, X., et al., Materials' tactile testing and characterisation for consumer products' affective packaging design. Materials \& Design, 2009. 30(10): p. 4299-4310.

30. Picard, D., et al., Perceptual dimensions of tactile textures. Acta Psychologica, 2003. 114(2): p. 165184.

31. Yoshioka, T., et al., Texture perception through direct and indirect touch: An analysis of perceptual space for tactile textures in two modes of exploration. Somatosensory And Motor Research, 2007. 24(1-2): p. 53-70.

32. Lyne, M., A. Whiteman, and D. Donderi, Multidimensional scaling of tissue quality. Pulp \& Paper Canada, 1984. 85(10): p. 43-50.

33. Hollins, M., et al., Individual differences in perceptual space for tactile textures: Evidence from multidimensional scaling. Perception \& Psychophysics, 2000. 62(8): p. 1534-1544.

34. Bergmann Tiest, W.M. and A.M.L. Kappers, Analysis of haptic perception of materials by multidimensional scaling and physical measurements of roughness and compressibility. Acta Psychologica, 2006. 121(1): p. 1-20.

35. Holliins, M., et al., Perceptual dimensions of tactile surface texture: A multidimensional scaling analysis. Perception \& psychophysics, 1993. 54(6): p. 697-705. 
36. Arvidsson, M., Getting a feel for tactile space: Exploring haptic perception of microtextures. 2012, Stockholm Univerity: Stockholm.

37. Skedung, L., Tactile perception: Role of Friction and Texture. 2012, KTH Royal Institute of Technology: Stockholm.

38. LaMotte, R.H. and M.A. Srinivasan, Surface Microgeometry: Tactile Perception and Neural Encoding Wenner-Gren International Symposium Series ed. Information Processing in the Somatosensory System, ed. O. Franzen and J. Westman. Vol. 4. 1991: MacMillan Press.

39. Bergmann Tiest, W.M., Tactual perception of material properties. Vision Research, 2010. 50(24): p. 2775-2782.

40. Blake, D.T., S.S. Hsiao, and K.O. Johnson, Neural coding mechanisms in tactile pattern recognition: The relative contributions of slowly and rapidly adapting mechanoreceptors to perceived roughness. Journal of Neuroscience, 1997. 17(19): p. 7480-7489.

41. Connor, C.E., et al., Tactile roughness: Neural codes that account for psychophysical magnitude estimates. Journal of Neuroscience, 1990. 10(12): p. 3823-3836.

42. Connor, C.E. and K.O. Johnson, Neural coding of tactile texture: Comparison of spatial and temporal mechanisms for roughness perception. Journal of Neuroscience, 1992. 12(9): p. 34143426.

43. Bensmaia, S. and M. Hollins, Pacinian representations of fine surface texture. Perception \& Psychophysics, 2005. 67(5): p. 842-854.

44. Bensmaia, S.J. and M. Hollins, The vibrations of texture. Somatosensory And Motor Research, 2003. 20(1): p. 33-43.

45. Arvidsson, M., Getting a Feel for Tactile Space: Exploring Haptic Perception of Microtexture. 2012.

46. Weber, A.I., et al., Spatial and temporal codes mediate the tactile perception of natural textures PNAS, 2013. 110(40): p. 17107-17112.

47. Saal, H.P. and S.J. Bensmaia, Touch is a team effort: interplay of submodalities in cutaneous sensibility. Trends in Neurosciences, (0).

48. Smith, A.M. and S.H. Scott, Subjective scaling of smooth surface friction. Journal Of Neurophysiology, 1996. 75(5): p. 1957-1962.

49. Chung, J.Y., A.J. Nolte, and C.M. Stafford, Surface wrinkling: A versatile platform for measuring thin-film properties. Advanced Materials, 2011. 23(3): p. 349-368.

50. Schweikart, A. and A. Fery, Controlled wrinkling as a novel method for the fabrication of patterned surfaces. Microchimica Acta, 2009. 165(3-4): p. 249-263.

51. Duvefelt, K., et al., Model for contact between finger and sinusoidal plane to evaluate adhesion and deformation component of friction. Tribology International, 2014.

52. Westergaard, H., Bearing Pressures and Cra; $c] ;<$ si. Journal of applied mechanics, 1939.

53. Pailler-Mattei, C., S. Bec, and H. Zahouani, In vivo measurements of the elastic mechanical properties of human skin by indentation tests. Medical engineering \& physics, 2008. 30(5): p. 599606.

54. Lees, C., J. Vincent, and J.E. Hillerton, Poisson's ratio in skin. Bio-medical materials and engineering, 1990. 1(1): p. 19-23.

55. Stevens, S.S., On the psychophysical law. Psychological review, 1957. 64(3): p. 153.

56. Gescheider, G.A., Psychophysics: the fundamentals. 2013: Psychology Press.

57. Andre, T., P. Lefevre, and J.-L. Thonnard, A continuous measure of fingertip friction during precision grip. Journal Of Neuroscience Methods, 2009. 179(2): p. 224-229.

58. André, T., P. Lefevre, and J.-L. Thonnard, Fingertip moisture is optimally modulated during object manipulation. Journal of neurophysiology, 2010. 103(1): p. 402-408.

59. André, T., et al., Effect of skin hydration on the dynamics of fingertip gripping contact. Journal of The Royal Society Interface, 2011. 8(64): p. 1574-1583. 
60. Álvarez-Asencio, R., et al., Nanomechanical properties of human skin and introduction of a novel hair indenter. Journal of the Mechanical Behavior of Biomedical Materials, 2016. 54: p. 185-193.

61. Gueorguiev, D., et al., Touch uses frictional cues to discriminate flat materials. Scientific reports, 2016. 6. 